\title{
Endemic Kaposi sarcoma in HIV-negative children and adolescents: an evaluation of overlapping and distinct clinical features in comparison with HIV-related disease
}

Nader Kim El-Mallawany ${ }^{1,2^{*}}$ D, Jimmy Villiera ${ }^{3,4}$, William Kamiyango ${ }^{3,4}$, Erin C. Peckham-Gregory ${ }^{1,2}$,

Michael E. Scheurer ${ }^{1,2}$, Carl E. Allen ${ }^{1,2}$, Casey L. McAtee ${ }^{1,2}$, Alejandra Legarreta ${ }^{1,2}$, Dirk P. Dittmer ${ }^{5}$, Carrie L. Kovarik ${ }^{6}$, Elizabeth Y. Chiao ${ }^{7,8}$, Stephen C. Martin ${ }^{1,2,3}$, Nmazuo W. Ozuah ${ }^{1,2}$, Parth S. Mehta ${ }^{1,2}$ and Peter N. Kazembe 3,4

\begin{abstract}
Background: Endemic Kaposi sarcoma (KS) was first described in African children over fifty years ago, but has recently been overshadowed by HIV-related disease. We aimed to evaluate the similarities and differences between endemic HIV-negative and epidemic HIV-positive pediatric KS in a KS-associated herpesvirus-endemic region of Africa.

Methods: We describe clinical characteristics of $20 \mathrm{HIV}$-negative children with endemic KS over a six-year period and compare findings with a historical control-an HIV-related pediatric KS cohort from Lilongwe, Malawi.

Results: The HIV-negative endemic KS cohort was 70\% male with a median age of 9.3 years. Lymph node involvement was present in 50\%, hyperpigmented skin lesions in 45\%, and woody edema in 40\%. One patient (5\%) presented with oral KS involvement and no patients presented initially with visceral KS. Significant anemia (hemoglobin $<8 \mathrm{~g} / \mathrm{dL}$ ) and thrombocytopenia (platelet count $<100 \times 10^{9} / \mathrm{L}$ ) were found at time of original KS diagnosis in 45 and 40\% respectively. In both HIV-negative and HIV-positive cohorts, lymphadenopathy was the most common presentation, prototypical skin lesions were often absent, severe cytopenias were a common clinical feature, and treatment outcomes were similar. Patients with endemic KS demonstrated less frequent oral involvement ( $5 \%$ versus $29 \%, P=0.03$ ) and a lower proportion of patients with visceral involvement ( $0 \%$ versus $16 \%, P=0.06)$.
\end{abstract}

Conclusions: These data suggest clinical overlap between epidemiological variants. Treatment protocols for pediatric KS in sub-Saharan Africa should be devised to include both endemic HIV-negative and epidemic HIV-related disease to better define the clinical and biological comparison.

Keywords: Kaposi sarcoma, Human herpesvirus-8, KS, KSHV, HHV-8, Endemic, HIV-negative, Pediatric oncology, Africa, Global health

\footnotetext{
* Correspondence: nader.el-mallawany@bcm.edu

${ }^{1}$ Baylor College of Medicine, Department of Pediatrics, Section of Hematology-Oncology, Houston, TX, USA

${ }^{2}$ Texas Children's Cancer and Hematology Centers, Baylor College of Medicine, Global HOPE (Hematology-Oncology Pediatric Excellence), 1102

Bates Street, Feigin Tower, Suite 1025.16, Houston, TX 77030, USA

Full list of author information is available at the end of the article
}

(c) The Author(s). 2018 Open Access This article is distributed under the terms of the Creative Commons Attribution 4.0 International License (http://creativecommons.org/licenses/by/4.0/), which permits unrestricted use, distribution, and reproduction in any medium, provided you give appropriate credit to the original author(s) and the source, provide a link to the Creative Commons license, and indicate if changes were made. The Creative Commons Public Domain Dedication waiver (http://creativecommons.org/publicdomain/zero/1.0/) applies to the data made available in this article, unless otherwise stated. 


\section{Introduction}

Descriptions of endemic Kaposi sarcoma (KS) of childhood originated more than fifty years ago in central and eastern Africa-decades before the emergence of HIV-offering details of the clinical characteristics and outcomes in HIV-negative African children living in regions with endemic human herpesvirus-8/KS-associated herpesvirus (KSHV) infection [1-5]. Unique features of endemic pediatric KS that were distinct from adults included frequent presentation primarily with lymph node disease, less frequent skin involvement, and a fulminant clinical course if untreated-especially for children with lymphadenopathic KS [3, 4].

Following those initial descriptions, little has been published on endemic HIV-unrelated KS [6-9]. With the alarming rise in HIV infection rates across sub-Saharan Africa over the past 25 years, the spotlight has shifted towards HIV-related KS. Increased efforts to treat pediatric $\mathrm{HIV}$ infection and its complications in sub-Saharan Africa have resulted in greater awareness and understanding of HIV-related KS in children [6, 7, 10-17]. Clinical patterns of HIV-related pediatric KS appear to overlap with the early observations of endemic KS from fifty years ago. The same unique features of predilection for lymph node involvement and its characteristic association with fulminant disease progression have distinguished it from $\mathrm{KS}$ in HIV-infected adults [11, 12, 14-16, 18].

In this brief report, we describe the clinical features and treatment outcomes of endemic pediatric $\mathrm{KS}$ and compare it to our previously published experience with HIV-related pediatric KS in Lilongwe, Malawi.

\section{Methods}

Medical records of HIV-negative children and adolescents $(<18$ years) with a histological or clinical diagnosis of endemic KS between January 2011 and December 2016 were reviewed. HIV status was determined based upon standard clinical procedure using rapid antibody testing and all patients underwent repeat testing for confirmation to rule-out the potential for a false-negative result. The initial ten patients also underwent qualitative HIV DNA PCR testing for additional confirmation of their HIV-negative status. Diagnostic and therapeutic algorithms have been previously described, including supportive care strategies [16]. In brief, first line chemotherapy was a combination of bleomycin and vincristine (BV); patients with relapsed/refractory disease received doxorubicin plus BV, and the third-line chemotherapy option was paclitaxel.

Patients were staged according to the Lilongwe Pediatric KS Staging Classification, a pediatric-specific staging paradigm that attempts to address the unique clinical features of childhood KS observed in KSHV-endemic regions of Africa $[17,19]$. Briefly, this staging classification differentiates four distinct clinical phenotypes with contrasting disease characteristics and treatment outcomes. Stage 1 includes patients with mild (stage 1A) to moderate (stage 1B) disease limited to the skin, subcutaneous tissue, oral mucosa, and conjunctiva. Stage 2 is defined as lymphadenopathic KS, while stage 3 is characterized by the presence of woody edema. Stage 4 is the group with the worst long-term survival and includes patients with visceral disease and those with 20 or more hyperpigmented skin/oral lesions in widespread distribution [17, 19].

Data from endemic KS patients were compared to data from a previously described cohort of HIV-related pediatric $\mathrm{KS}$ at the same institution [16]. All statistical analyses were performed using STATA v.13.1, and P-values were generated using either a $k$-sample equality-of-medians test, Chi-square test, or Fisher's exact test, as appropriate. The study was approved by the Malawi National Health Sciences Research Committee and the Baylor College of Medicine Institutional Review Board for Human Subjects; a waiver of consent was obtained due to the retrospective nature of the study.

\section{Results \\ Clinical characteristics of endemic pediatric KS}

There were 20 children ( $70 \%$ male) diagnosed with endemic KS between 2011 and 2016. In comparison, there were 140 new HIV-related pediatric KS diagnoses over the same six-year time period [20]. Additional file 1: Figure S1 demonstrates a comparison of the histopathological appearance of lymphadenopathic KS in both endemic and HIV-related KS. The most common clinical site of endemic KS involvement was lymph node (50\%) followed by hyperpigmented skin lesions (45\%) and woody edema (40\%, Table 1). No patient had visceral involvement on original presentation and one (5\%) presented with oral involvement. Nine (45\%) patients presented with moderate-severe anemia (hemoglobin $<8 \mathrm{~g} / \mathrm{dL}$ ) and 8 (40\%) with moderate-severe thrombocytopenia (platelet count $\left.<100 \times 10^{9} / \mathrm{L}\right)$.

At a median follow-up of 39 months (interquartile range 27-62), 13 patients (65\%) were alive. Nine (45\%) were in complete remission (CR, defined as complete and sustained disappearance of all KS lesions), 3 (15\%) were alive with stable disease, and 1 patient had experienced relapse and was receiving salvage chemotherapy. Seven (35\%) patients died at median time of 2 months (range 0.1-52) from the date of KS diagnosis. Six of those deaths were attributed to $\mathrm{KS}$ and there were no treatment-related deaths.

\section{Comparison of endemic and HIV-related pediatric KS}

Comparing this series of 20 children with endemic KS to our previously published cohort of 70 children with HIV-related KS, some similarities and differences emerged (Table 1). 
Table 1 Clinical Characteristics of Children with Endemic HIV-Negative KS vs HIV-Related KS

\begin{tabular}{|c|c|c|c|}
\hline Epidemiologic subtype of kaposi sarcoma & Endemic & HIV-related & $p$ \\
\hline Number of Patients & 20 & 70 & \\
\hline Number of Females & $6(30 \%)$ & $35(50 \%)$ & $0.11^{\mathrm{a}}$ \\
\hline Median Age in years (range) & $9.3(2.0-16.3)$ & $8.4(1.7-17.9)$ & $0.57^{b}$ \\
\hline Pathology Confirmation & $8(40 \%)$ & $14(20 \%)$ & $0.07^{\mathrm{a}}$ \\
\hline \multicolumn{4}{|l|}{ Clinical Site(s) of KS Involvement } \\
\hline Lymph Node & $10(50 \%)$ & $52(74 \%)$ & $0.03^{\mathrm{a}}$ \\
\hline Hyperpigmented Skin Lesions & $9(45 \%)$ & $42(60 \%)$ & $0.21^{\mathrm{a}}$ \\
\hline Woody Edema & $8(40 \%)$ & $17(24 \%)$ & $0.18^{\mathrm{a}}$ \\
\hline Flesh Colored Subcutaneous Nodules & $7(35 \%)$ & $23(33 \%)$ & $0.89^{a}$ \\
\hline Oral & $1(5 \%)$ & $20(29 \%)$ & $0.03^{c}$ \\
\hline Lymph Node ONLY & $5(25 \%)$ & $18(26 \%)$ & $0.95^{\mathrm{a}}$ \\
\hline Visceral & 0 & $11(16 \%)$ & $0.06^{\mathrm{C}}$ \\
\hline Disseminated (ie $\geq 20$ ) Hyperpigmented Skin/Oral Lesions & $4(20 \%)$ & $7(10 \%)$ & $0.26^{\mathrm{C}}$ \\
\hline Anemia (hemoglobin < $<$ ) at KS diagnosis & $9(45 \%)$ & $25(37 \%) *$ & $0.54^{\mathrm{a}}$ \\
\hline Thrombocytopenia (platelet count $<100$ ) at KS diagnosis & $8(40 \%)$ & $19(28 \%) *$ & $0.32^{\mathrm{a}}$ \\
\hline \multicolumn{4}{|l|}{ Treatment Outcome } \\
\hline Alive in Complete Remission & $9(45 \%)$ & $33(47 \%)$ & $0.31^{\mathrm{C}}$ \\
\hline Alive with Stable Disease & $4(20 \%)$ & $6(9 \%)$ & \\
\hline Death & $7(35 \%)$ & $31(44 \%)$ & \\
\hline \multicolumn{4}{|l|}{ Lilongwe Pediatric KS Staging Classification* } \\
\hline Stage 1A (mild cutaneous/oral KS) & 0 & 0 & $0.08^{\mathrm{C}}$ \\
\hline Stage 1B (moderate cutaneous/oral KS) & $4(20 \%)$ & $3(4 \%)$ & \\
\hline Stage 2 (lymphadenopathic KS) & $6(30 \%)$ & $34(50 \%)$ & \\
\hline Stage 3 (woody edema KS) & $6(30 \%)$ & $14(21 \%)$ & \\
\hline Stage 4 (visceral and/or disseminated KS) & $4(20 \%)$ & $17(25 \%)$ & \\
\hline
\end{tabular}

Legend: KS Kaposi sarcoma, ${ }^{*}$ in the HIV-related KS cohort, 67 patients had baseline blood count assessments and 68 patients were fully staged. ${ }^{2}$-value estimated using the Chi-square test $;{ }^{b}$ Chi-square $P$-value estimated from the $\mathrm{k}$-sample equality of medians test ${ }^{;} P$-value estimated using the Fisher's exact test

In both cohorts, KS lymphadenopathy was the most common presenting feature, although this occurred more frequently among HIV-related KS cases $(P=0.03)$. Approximately one-fourth of both cohorts presented with KS lymphadenopathy in the absence of prototypical skin/oral lesions or woody edema $(P=0.95)$. Hyperpigmented skin lesions were present in around half of both cohorts $(P=0.21)$. Moderate-severe anemia and/ or thrombocytopenia commonly occurred in both epidemiological variants $(P=0.54$ and $P=0.32$, respectively).

Distinctions between endemic and HIV-related KS were demonstrated as well. Patients with endemic KS had a lower frequency of oral KS involvement $(P=0.03)$ compared to the HIV-related control group. None of the endemic KS patients originally presented with visceral disease compared to $16 \%$ of HIV-related KS patients $(P=0.06)$, and the endemic KS cohort was $70 \%$ male compared to $50 \%$ in the HIV-related KS cohort $(P=0.11)$. Ultimately, treatment outcomes were similar $(P=0.31)$.

\section{Treatment outcomes}

Nineteen patients were initiated on the chemotherapy regimen described above, with one patient having died prior to initiation of chemotherapy. Sixteen patients completed the treatment protocol and 3 died from refractory KS that progressed despite chemotherapy. Survival in context of the Lilongwe Pediatric KS Staging Classification was explored (Table 2) [17, 19]. In summary, 8 of 10 patients $(80 \%)$ with stage I/II KS were alive in CR with follow-up ranging 14-62 months from time of KS diagnosis. Among the 10 patients with stage III/IV KS, only one $(10 \%)$ was alive in CR. Five $(50 \%)$ patients with stage III/IV disease died from relapsed/refractory KS, while $4(40 \%)$ were alive with stable disease.

Mortality patterns were categorized in context of the pediatric-specific staging paradigm as well (Table 2). No patient fit criteria for stage 1A (mild cutaneous/oral KS). Of the 4 patients categorized as stage $1 \mathrm{~B}$ (moderate cutaneous/oral KS), there was one death, occurring in a patient who presented concurrently with KS (diagnosed 
Table 2 Characteristics of Children and Adolescents with Endemic KS in Context of a Pediatric-Specific Staging Classification

\begin{tabular}{lllll}
\hline & $\begin{array}{l}\text { Stage 1: Mild/Moderate } \\
\text { Cutaneous/Oral KS }\end{array}$ & Stage 2: Lymphadenopathic KS & $\begin{array}{l}\text { Stage 3: Woody } \\
\text { Edema KS }\end{array}$ & $\begin{array}{l}\text { Stage 4: Visceral and/or } \\
\text { Disseminated Cutaneous/ } \\
\text { Oral KS }\end{array}$ \\
\hline Number of patients, $n$ & 4 & 6 & 6 & 4 \\
Baseline Anemia (hgb $<8), n(\%)$ & 0 & $5(83 \%)$ & $1(17 \%)$ & $3(75 \%)$ \\
$\begin{array}{l}\text { Baseline Thrombocytopenia } \\
\text { (platelet count }<100), n(\%)\end{array}$ & $1(25 \%)$ & $4(67 \%)$ & 0 & $3(75 \%)$ \\
Alive in complete remission, $n(\%)$ & $3(75 \%)$ & $5(83 \%)$ & $1(17 \%)$ & 0 \\
Alive with stable disease, $n(\%)$ & 0 & 0 & $3(50 \%)$ & $1(25 \%)$ \\
Deaths, $n(\%)$ & $1(25 \%)$ & $1(17 \%)$ & $2(33 \%)$ & $3(75 \%)$ \\
$\quad$ KS related death & 0 & 1 & 2 & 3 \\
$\quad$ Non-KS related death & 1 & 0 & 0 & 0 \\
2-year EFS in HIV+ KS cohort ${ }^{\mathrm{a}}$ & too few patients to determine & $73 \%$ & $29 \%$ & 0 \\
2-year OS in HIV+ KS cohort ${ }^{2}$ & & $75 \%$ & $79 \%$ & $12 \%$ \\
\hline
\end{tabular}

Legend: KS Kaposi sarcoma, $h g b$ hemoglobin, EFS event-free survival, OS overall survival, ${ }^{a}$ references the published historical HIV-related pediatric KS cohort (reference number 17)

clinically with prototypical skin and conjunctival eye lesions) and histologically-confirmed Hodgkin lymphoma. This patient died of refractory and progressive lymphoma; the KS lesions had completely resolved. Among the 6 patients in stage 2 (lymphadenopathic KS), there was also one death, occurring secondary to severe cytopenias on the first day of hospitalization, before chemotherapy could be initiated.

Of the 6 patients categorized as stage 3 (woody edema $\mathrm{KS}), 1$ was in CR, 3 were alive with stable disease off chemotherapy, and 2 had died. One death occurred in a child that was lost to follow-up after six months of chemotherapy and was subsequently found to have died one year later, via telephone communication. The other death occurred in a child that originally presented with woody edema, hyperpigmented skin lesions, and lymphadenopathy, eventually evolving with the second relapse to include pulmonary visceral involvement with severe serosanguineous pleural effusions. All 4 of the patients categorized as stage 4 originally presented with disseminated cutaneous/oral KS (widespread distribution of $\geq 20$ hyperpigmented skin/oral lesions) and without visceral involvement. Three of them were refractory to chemotherapy and their underlying KS rapidly progressed (time of death ranged 0.5-2 months). One patient achieved CR with up-front intensified chemotherapy (doxorubicin plus BV), however eventually experienced relapse 34 months after the original diagnosis and was alive with stable disease but receiving salvage chemotherapy with paclitaxel at the time of data analysis.

\section{Discussion}

This cohort of HIV-negative children and adolescents with endemic KS shows considerable overlap with HIV-related disease. Primary presentation with lymphadenopathy was a consistent clinical feature in both pediatric endemic and HIV-related KS cohorts. Additionally, in both epidemiological variants, presentation with severe cytopenias is frequent and approximately half of the patients lacked the prototypical hyperpigmented skin/oral lesions that are so often relied upon as a clinical indicator of KS. Distinctions between endemic and HIV-related pediatric KS were also observed, as we noted the uncommon occurrence of oral lesions in HIV-negative patients, as well as a lower frequency of visceral involvement in endemic KS.

These findings are consistent with previously published reports on endemic KS in Africa (Table 3). The predilection for lymph node involvement in both endemic and HIV-related KS emphasizes the importance of recognizing $\mathrm{KS}$ as an important disease in the differential diagnosis for children with persistent lymphadenopathy in KSHV-endemic regions of Africa. Our observation of infrequent oral involvement in endemic KS has also been previously described and ranges from 0 to $10 \%$ in published historical cohorts from across the region (Table 3). This contrasts with HIV-related pediatric KS cohorts in the same region, in which oral KS lesions have been reported in $21-58 \%$ of patients [19]. It is more challenging to confirm the true incidence of visceral disease in endemic KS because of limitations in resources required to confidently establish the diagnosis in low-income countries. Additionally, there appeared to be a male predominance in endemic KS, although similar to the comparison of visceral involvement, this failed to meet statistical significance. Historically, endemic KS cohorts ranged from 70 to $84 \%$ male in publications including at least 20 subjects, while male representation in HIV-related pediatric KS has ranged from 50 to $73 \%$ [19]. While these data, as well as the historical precedents are limited by sample size, it is 
Table 3 Comparison of Clinical Features Among Endemic HIV-Negative Pediatric KS Cohorts in Africa

\begin{tabular}{|c|c|c|c|c|c|c|c|c|c|}
\hline \multirow[t]{2}{*}{ Location(s) } & \multirow{2}{*}{$\begin{array}{l}\text { Time } \\
\text { Period }\end{array}$} & \multirow[t]{2}{*}{$n$} & \multirow[t]{2}{*}{ Age of Cohort } & \multirow{2}{*}{$\begin{array}{l}\% \text { Male } \\
\text { Gender }\end{array}$} & \multicolumn{5}{|c|}{ Original Clinical Presentation with KS Involvement of: } \\
\hline & & & & & Lymph Node & Skin Lesions & Woody Edema & Oral Lesions & Visceral Disease \\
\hline Uganda/Tanzania $^{3}$ & $1957-65$ & 51 & median 10 years & $76 \%$ & $53 \%$ & $53 \%$ & no mention & no mention & no mention \\
\hline Uganda ${ }^{4}$ & 1968-75 & 12 & median 8 years & $58 \%$ & $83 \%$ & $17 \%$ & no mention & $8 \%$ & $8 \%$ \\
\hline Tanzania $^{6}$ & $1968-82$ & 73 & $63 \%$ under 5 years & $84 \%$ & $48 \%$ & uncertain & no mention & $1 \%$ & no mention \\
\hline Kenya $^{7}$ & 1997-99 & 8 & median 7 years & $88 \%$ & $75 \%$ & $38 \%$ & uncertain & 0 & $13 \%$ \\
\hline Blantyre, Malawi ${ }^{8}$ & $2002-14$ & 20 & median 4 years & $80 \%$ & $60 \%$ & $40 \%$ & $70 \%$ & $10 \%$ & uncertain \\
\hline Lilongwe, Malawi & 2011-16 & 20 & median 9 years & $70 \%$ & $50 \%$ & $45 \%$ & $40 \%$ & $5 \%$ & 0 \\
\hline
\end{tabular}

Legend: $K S$ Kaposi sarcoma, no mention = indicates that these data were not mentioned at all in the publication, uncertain = indicates that although the topic was mentioned in the publication, discrete numbers were not reported. Associated references are listed in superscript in the first column

plausible that subtle distinctions between the two epidemiological variants of pediatric KS in Africa exist.

Reviewing HIV-related parameters of the HIV-infected pediatric KS cohort, a few important observations are noted that may explain the clinical overlap with endemic disease. Severe CD4 count suppression was infrequent, observed in only $28 \%$ of the HIV-related cohort [16]. Additionally, 49\% of the HIV-related cohort was already on antiretroviral therapy at the time of KS diagnosis, half of them for more than 12 months [16]. Considering the relatively high proportion of HIV-infected children developing KS despite having immunocompetent CD4 counts and/or on antiretroviral therapy, it appears that the immunologic context in which HIV-related and endemic KS occurs is often similar, and potentially more attributed to an undefined qualitative immune dysfunction rather than a severe quantitative suppression of the CD4 count. The authors hypothesize that this may provide rationale for the often-overlapping clinical features observed, and may even become more apparent in the future with increasing access to antiretroviral therapy.

The occurrence of moderate-severe anemia and thrombocytopenia as a presenting clinical feature of pediatric KS has been consistent in the experiences with both HIV-negative and HIV-related disease in Malawi. Although numbers are too small in this cohort to evaluate clinical associations, presentation with cytopenias was not found to have an impact on survival in HIV-related pediatric KS [16]. Baseline cytopenias were significantly more likely to occur in HIV-infected children with stage 2 lymphadenopathic KS and stage 4 visceral and/ or disseminated cutaneous/oral KS, and this pattern appears to be consistent in our experience with endemic KS as well (Table 2) [17].

Considering the frequent occurrence of cytopenias as well as the overlapping clinical patterns of disease in both epidemiological variants of pediatric KS, it is plausible that KSHV-driven pathways may play an important biological role in determining the clinical phenotype of disease. Recent pilot data from HIV-infected children in Malawi demonstrated associations between elevated plasma KSHV viral load and lymphadenopathic KS, and although sample size limited the statistical power of the results, it was notable that all 5 patients with anemia as well as all 4 patients with thrombocytopenia demonstrated detectable KSHV viral loads at the time of KS diagnosis [21]. If the clinical features are driven by KSHV-mediated pathways, they may be more attributed to mechanisms of KSHV pathogenesis, and therefore less dependent on the underlying HIV-status. Additionally, elevated interleukin-6 (IL-6) levels were common in the pilot pediatric cohort, and the links between lytic activation of KSHV, elevated viral load and IL-6 levels, and clinical presentation with cytopenias have been well established in adults with other KSHV-associated malignancies including multicentric Castleman disease (MCD) and the KSHV inflammatory cytokine syndrome (KICS) [21, 22]. Based on this, the authors hypothesize that analogous to the KSHV-driven viral pathophysiology in MCD and KICS, lytic activation of KSHV in subsets of both HIV-positive and HIV-negative pediatric KS patients may be linked with specific clinical findings (eg KS lymphadenopathy, visceral/disseminated disease, and the presence of anemia and thrombocytopenia). Ultimately, biological assessments of both epidemiological variants are required to establish the precise pathophysiological mechanisms driving the distinct clinical features that have been observed.

Cancer registry data and GLOBOCAN estimates demonstrate that alongside lymphoma (including Burkitt, Hodgkin, and non-Burkitt non-Hodgkin lymphoma), Wilms tumor, retinoblastoma, and leukemia, KS has become one of the top five most common overall childhood malignancies in several countries in eastern and central Africa including Malawi, Uganda, Kenya, Tanzania, Rwanda, Mozambique, Zambia, and Zimbabwe [23]. Contemporary pediatric KS publications from this region though, have mostly focused on HIV-infected patients. Although rates of HIV-related KS exceed those of endemic disease in this era of the HIV epidemic, it is important to establish a treatment paradigm that includes children with endemic KS as well [9]. Observations from this study suggest that overall outcomes between endemic 
and HIV-related cohorts are similar in the context of readily available antiretroviral therapy for HIV-infected patients. Although there are not enough patients in this analysis to draw definitive conclusions, prognosis appears to correlate more with the clinical phenotype of disease presentation (ie their classification in a pediatric-specific risk-stratification platform) rather than the underlying epidemiological classification of the disease.

Ultimately, it appears that there is significant overlap in the clinical features between endemic and HIV-related KS in children and adolescents in a KSHV-endemic region of Africa. The authors advocate for a universal risk-stratified treatment approach that applies to both endemic and HIV-related pediatric KS. Systematically characterizing the treatment response for children with endemic disease will be essential to improving overall outcomes.

\section{Additional file}

Additional file 1: Figure S1. Lymph Node Histopathology in Patients with Endemic HIV-Negative and Epidemic HIV-Positive Lymphadenopathic Kaposi Sarcoma. Lymph node biopsies from endemic HIV-negative (A) and epidemic HIV-positive (B) children with lymphadenopathic Kaposi sarcoma demonstrating diffuse spindle cell tumor infiltrates that stain positive for the Kaposi sarcoma herpesvirus/human herpesvirus-8 latency-associated nuclear antigen immunohistochemical stain at 200x magnification. (TIF $7072 \mathrm{~kb}$ )

\section{Abbreviations \\ BV: Bleomycin and vincristine; CR: Complete remission; EFS: Event-free survival; IL-6: Interleukin-6; KICS: KSHV inflammatory cytokine syndrome; KS: Kaposi sarcoma; KSHV: Kaposi sarcoma-associated herpesvirus; MCD: Multicentric Castleman disease; OS: Overall survival}

\section{Acknowledgements}

We express admiration and gratitude to our patients and their families, fighting a brave battle against severe illness in the setting of severe limitations in societal infrastructure and medical resources. We thank our many colleagues at the Baylor College of Medicine Children's Foundation Malawi inclusive of the Tingathe Outreach Program, the Baylor International Pediatric AIDS Initiative at Texas Children's Hospital, the Texas Children's Cancer and Hematology Centers and the Global HOPE (HematologyOncology Pediatric Excllence) Program, and Kamuzu Central Hospital with particular appreciation for the Pathology Laboratory and the Department of Paediatrics. We especially wish to thank Brent Eason from the University of North Carolina Vironomics Core Laboratory for the pathology images highlighting the immunohistochemical findings of pediatric KS.

\section{Funding}

Research reported in this publication was supported by R21CA217137 as well as public health service grants CA019014, CA190152, and CA192744. The clinical work of the pediatric HIV-related malignancy program at the Baylor College of Medicine Children's Foundation Malawi and Kamuzu Central Hospital in Lilongwe, Malawi was supported in part by a grant from the United States Agency for International Development through the Tingathe Program via cooperative agreement number 674-A-00-10-00093-00 and philanthropic contributions to purchase chemotherapy from ConocoPhillips. The content is solely the responsibility of the authors and does not necessarily represent the official views of the National Institutes of Health or the other funders. The funders had no role in study design, data collection and analysis, decision to publish, or preparation of the manuscript.

\section{Availability of data and materials}

The dataset has been uploaded to Dryad at the following DOl: https:// doi.org/10.5061/dryad.75tn6. Any additional data may be requested from the corresponding author.

\section{Authors' contributions}

Conceived and designed the experiments: NKE JV WK MES PSM PNK. Collection and assembly of data: NKE JV WK AL. Analyzed and interpreted the data: NKE ECPG MES CEA CLM DPD CLK EYC SCM NWO. Contributed reagents/materials/analysis tools: NKE ECPG MES DPD CLK. All authors contributed to the manuscript writing, read and approved the final version.

\section{Ethics approval and consent to participate}

Ethics committee approval was obtained from the Malawi National Health Science Research Committee and Baylor College of Medicine Institutional Review Board for Human Subjects Research. Due to the retrospective nature of this study, a waiver of consent was obtained. All data were anonymized and de-identified prior to analysis to maintain strict protection of confidentiality.

\section{Consent for publication}

Not applicable.

\section{Competing interests}

The authors have no competing interests to declare.

\section{Publisher's Note}

Springer Nature remains neutral with regard to jurisdictional claims in published maps and institutional affiliations.

\section{Author details \\ ${ }^{1}$ Baylor College of Medicine, Department of Pediatrics, Section of Hematology-Oncology, Houston, TX, USA. ${ }^{2}$ Texas Children's Cancer and Hematology Centers, Baylor College of Medicine, Global HOPE (Hematology-Oncology Pediatric Excellence), 1102 Bates Street, Feigin Tower, Suite 1025.16, Houston, TX 77030, USA. ${ }^{3}$ Baylor College of Medicine Children's Foundation Malawi, Lilongwe, Malawi. ${ }^{4}$ Kamuzu Central Hospital, Lilongwe, Malawi. ${ }^{5}$ Lineberger Comprehensive Cancer Center, University of North Carolina, Chapel Hill, NC, USA. 'University of Pennsylvania, Philadelphia, PA, USA. ${ }^{7}$ Center for Innovation, Quality, Effectiveness and Safety (IQuESt), Michael E. DeBakey VA Medical Center, Houston, TX, USA. ${ }^{8}$ Baylor College of Medicine, Department of Medicine, Section of Infectious Diseases, Houston, TX, USA.}

Received: 16 August 2018 Accepted: 31 October 2018

Published online: 09 November 2018

References

1. Dutz W, Stout AP. Kaposi's sarcoma in infants and children. Cancer. 1960;13: 684-94

2. Davies JN, Lothe F. Kaposi's sarcoma in African children. Acta Unio Int Contra Cancrum. 1962;18:394-9.

3. Slavin G, Cameron HM, Forbes C, Mitchell RM. Kaposi's sarcoma in east African children: a report of 51 cases. J Pathol. 1970;100(3):187-99.

4. Olweny CL, Kaddumukasa A, Atine I, Owor R, Magrath I, Ziegler JL. Childhood Kaposi's sarcoma: clinical features and therapy. Br J Cancer. 1976; 33(5):555-60.

5. Tasque, Journiac, Dzietham. Kaposi's disease in a Cameroon infant aged 3 and a half years with macropolyadenopathies and death in 14th month. Sem Hop. 1955;31(75/8):429-30.

6. Amir H, Kaaya EE, Manji KP, Kwesigabo G, Biberfeld P. Kaposi's sarcoma before and during a human immunodeficiency virus epidemic in Tanzanian children. Pediatr Infect Dis J. 2001;20(5):518-21.

7. Mwanda OW, Fu P, Collea R, Whalen C, Remick SC. Kaposi's sarcoma in patients with and without human immunodeficiency virus infection, in a tertiary referral Centre in Kenya. Ann Trop Med Parasitol. 2005;99(1):81-91.

8. Mittermayer-Vassallo K, Banda K, Molyneux EM. Kaposi sarcoma in HIVseronegative children presenting to the paediatric oncology ward in the Queen Elizabeth central hospital, Blantyre, Malawi during 2002-2014. Trop Dr. 2016;46(3):138-42. 
9. Host KM, Horner MJ, van der Gronde T, Moses A, Phiri S, Dittmer DP, et al. Kaposi's sarcoma in Malawi: a continued problem for HIV-positive and HIVnegative individuals. AIDS. 2017;31(2):318-9.

10. Ziegler JL, Katongole-Mbidde E. Kaposi's sarcoma in childhood: an analysis of 100 cases from Uganda and relationship to HIV infection. Int J Cancer. 1996;65(2):200-3.

11. Gantt S, Kakuru A, Wald A, Walusansa V, Corey L, Casper C, et al. Clinical presentation and outcome of epidemic Kaposi sarcoma in Ugandan children. Pediatr Blood Cancer. 2010;54(5):670-4.

12. Vaz P, Macassa E, Jani I, Thome B, Mahagaja E, Madede T, et al. Treatment of Kaposi sarcoma in human immunodeficiency virus-1-infected Mozambican children with antiretroviral drugs and chemotherapy. Pediatr Infect Dis J. 2011;30(10):891-3.

13. Stefan DC, Stones DK, Wainwright L, Newton R. Kaposi sarcoma in south African children. Pediatr Blood Cancer. 2011;56(3):392-6.

14. Cox CM, El-Mallawany NK, Kabue M, Kovarik C, Schutze GE, Kazembe PN, et al. Clinical characteristics and outcomes of HIV-infected children diagnosed with Kaposi sarcoma in Malawi and Botswana. Pediatr Blood Cancer. 2013; 60(8):1274-80

15. Chagaluka G, Stanley C, Banda K, Depani S, Nijram'madzi J, Katangwe T, et al. Kaposi's sarcoma in children: an open randomised trial of vincristine, oral etoposide and a combination of vincristine and bleomycin. Eur J Cancer. 2014;50(8):1472-81.

16. El-Mallawany NK, Kamiyango W, Slone JS, Villiera J, Kovarik CL, Cox CM, et al. Clinical factors associated with long-term complete remission versus poor response to chemotherapy in HIV-infected children and adolescents with Kaposi sarcoma receiving bleomycin and vincristine: a retrospective observational study. PLoS One. 2016;11(4):e0153335.

17. El-Mallawany NK, Kamiyango W, Villiera J, Slone JS, Kovarik CL, Campbell LR, et al. Proposal of a risk-stratification platform to address distinct clinical features of pediatric Kaposi sarcoma in Lilongwe. Malawi J Glob Oncol. 2018:4:1-7.

18. Arkin $L M$, Cox CM, Kovarik CL. Kaposi's sarcoma in the pediatric population: the critical need for a tissue diagnosis. Pediatr Infect Dis J. 2009;28(5):426-8.

19. El-Mallawany NK, McAtee CL, Campbell LR, Kazembe PN. Pediatric Kaposi sarcoma in context of the HIV epidemic in sub-Saharan Africa: current perspectives. Pediatric Health Med Ther. 2018;9:35-46.

20. El-Mallawany NK, Villiera J, Kamiyango W, Mhango J, Slone JS, Mehta PS, et al. Increasing numbers of new Kaposi sarcoma diagnoses in HIV-infected children and adolescents despite the wide availability of antiretroviral therapy in Malawi. Clin Infect Dis. 2017;64(6):818-9.

21. El-Mallawany NK, Mehta PS, Kamiyango W, Villiera J, Peckham-Gregory EC, Kampani C, et al. KSHV viral load and Interleukin-6 in HIV-associated pediatric Kaposi sarcoma-exploring the role of lytic activation in driving the unique clinical features seen in endemic regions. Int J Cancer. 2018.

22. Polizzotto MN, Uldrick TS, Hu D, Yarchoan R. Clinical manifestations of Kaposi sarcoma herpesvirus lytic activation: multicentric Castleman disease (KSHV-MCD) and the KSHV inflammatory cytokine syndrome. Front Microbiol. 2012;3:73.

23. Kruger $M$, Hendricks $M$, Davidson A, Stefan CD, van Eyssen AL, Uys $R$, et al. Childhood cancer in Africa. Pediatr Blood Cancer. 2014;61(4):587-92.

\section{Ready to submit your research? Choose BMC and benefit from:}

- fast, convenient online submission

- thorough peer review by experienced researchers in your field

- rapid publication on acceptance

- support for research data, including large and complex data types

- gold Open Access which fosters wider collaboration and increased citations

- maximum visibility for your research: over $100 \mathrm{M}$ website views per year

At BMC, research is always in progress.

Learn more biomedcentral.com/submissions 